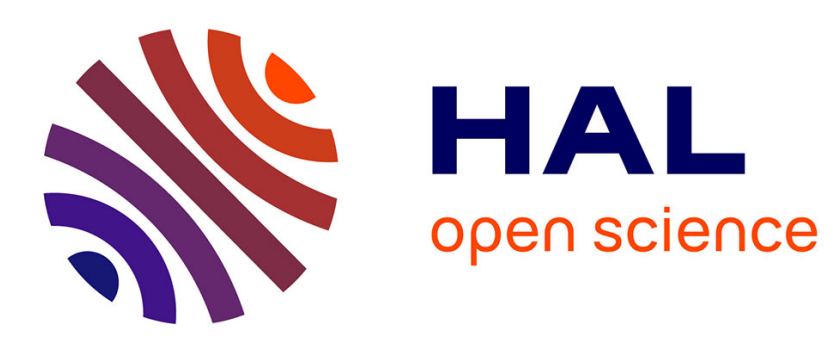

\title{
Nouvelle méthode de quantification des orientations cristallines par figures de pôles haute résolution et ultra-rapides
}

R. Fillit, F. Duchemin, J. Becker

\section{- To cite this version:}

R. Fillit, F. Duchemin, J. Becker. Nouvelle méthode de quantification des orientations cristallines par figures de pôles haute résolution et ultra-rapides. Journal de Physique IV Proceedings, 1996, 06 (C4), pp.C4-297-C4-306. 10.1051/jp4:1996428 . jpa-00254311

\section{HAL Id: jpa-00254311 https://hal.science/jpa-00254311}

Submitted on 1 Jan 1996

HAL is a multi-disciplinary open access archive for the deposit and dissemination of scientific research documents, whether they are published or not. The documents may come from teaching and research institutions in France or abroad, or from public or private research centers.
L'archive ouverte pluridisciplinaire HAL, est destinée au dépôt et à la diffusion de documents scientifiques de niveau recherche, publiés ou non, émanant des établissements d'enseignement et de recherche français ou étrangers, des laboratoires publics ou privés. 


\title{
Nouvelle méthode de quantification des orientations cristallines par figures de pôles haute résolution et ultra-rapides
}

\author{
R.Y. Fillit, F. Duchemin et J.M. Becker* \\ Ecole Nationale Supérieure des Mines de Saint-Etienne, URA 1884 du CNRS, 158 cours Fauriel, \\ 42023 Saint-Etienne cedex 2, France \\ * C.P.E Lyon, L.I.S.A., J.E 0091 C.N.R.S., 31 place Bellecour, 69006 Lyon, France
}

\begin{abstract}
Résumé : Afin d'améliorer la caractérisation des textures dans les matériaux nouveaux qui présentent des orientations cristallines toujours plus étroites (couches minces, macles, monocristaux, etc...), nous avons développé une méthode originale d'analyse à partir de figures de pôles $\mathrm{RX}$ à haute résolution angulaire et à grande vitesse d'acquisition. L'exploration angulaire est associée à un maillage hémisphérique adaptatif et un traitement des données de type image a été implémenté pour corriger les données instrumentales, puis interpréter et quantifier automatiquement les analyses. L'ensemble est relié à des calculs FDOC haute résolution du dispositif DOSOPHATEX.
\end{abstract}

\begin{abstract}
An improved method for texture characterization has been developed for heavy textured specimens (thin films, twins, single crystals, etc...), using a new high resolution XRay pole figure technique with a high speed measurement. An adaptive hemispheric scanning and a data treatment using digital image processing has been implemented for both diffractometer correction and automatic texture quantification. This method is linked to a new high resolution FDOC determination associated with the DOSOPHATEX system.
\end{abstract}

\section{INTRODUCTION}

Que ce soit pour l'étude des couches minces épitaxiques (diodes laser, couches minces supraconductrices, nanomatériaux, etc...) ou bien pour celle des mécanismes des déformations plastiques et des restaurations dynamiques (sur des monocristaux, des matériaux à gros grains, des maclages, etc..., soumis à des sollicitations mécaniques), il est fondamental d'extraire avec une grande précision les textures cristallographiques, c'est-à-dire les fractions volumiques des composantes des différentes orientations cristallines. Cependant, deux difficultés majeures apparaissent alors: premièrement, pour étudier ces orientations, on a besoin de figures de pôles très haute résolution $\left(0.01^{\circ} \times 0.01^{\circ}\right)$ [1], afin d'éviter la mauvaise détection de pics de diffraction extrêmement localisés et fins; deuxièmement, la détermination quantitative des fractions volumiques de ces orientations a été développée autour du calcul des FDOC [2], lesquelles s'appliquent généralement à des analyses de textures très basse résolution $\left(5^{\circ} \times 5^{\circ}\right)$ et présentent des erreurs (fantômes) dans le cas de textures très prononcées (les pôles étant très localisés); dans le cas de l'analyse par harmoniques sphériques généralisée, ces erreurs sont principalement dûes à la troncature des séries considérées. La méthode E.B.S.P d'analyse complémentaire des textures $[3,4]$ présente le grand avantage de permettre des déterminations locales mais a un double inconvénient : la précision angulaire est faible et la statistique réduite accrôt considérablement l'incertitude. 
Ainsi, dans un premier temps, nous avons développé une méthode de détermination de FDOC [5] utilisant non pas des figures de pôles standard $\left(5^{\circ} \times 5^{\circ}\right.$ en $\left.\varphi, \psi\right)$ à pas fixe, mais pouvant $s^{\prime}$ adapter automatiquement et continûment à des figures de pôles de plus haute résolution (jusqu'à $1^{\circ} \times 1^{\circ}$ ) (Fig. 1). Cette méthode originale conduit cependant à une utilisation d'une mémoire de plus en plus grande (croissance à la puissance 3 du nombre de pas angulaires mesurés).

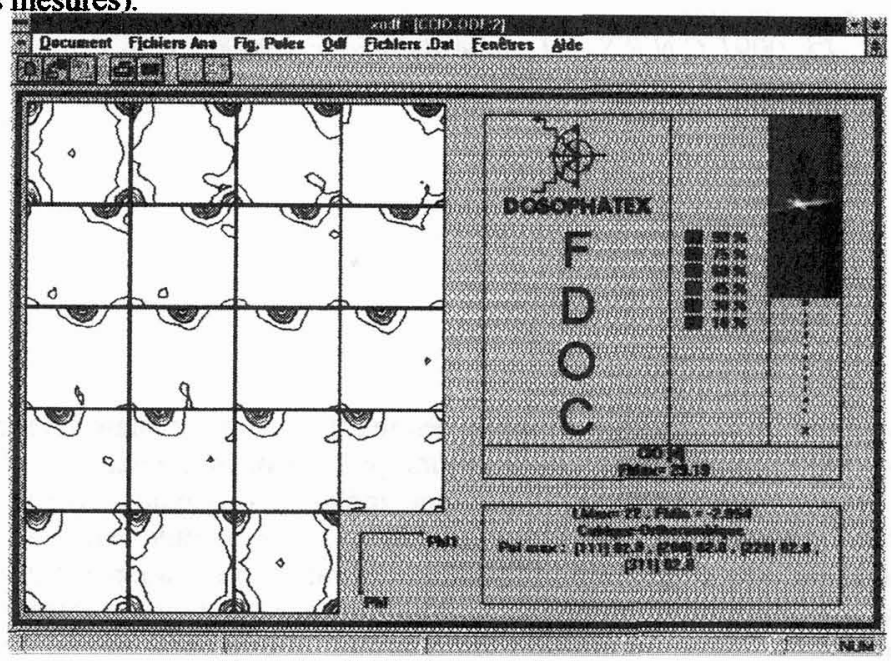

Fiqure 1: O.D.F. haute résolution

Pour pallier cette limite informatique, nous avons développé conjointement une nouvelle analyse par diffraction $X$ haute résolution et un traitement de données associé qui donne d'excellents résultats. Cette méthode de mesure s'avère particulièrement rapide (une dizaine de minutes environ), tout en générant des fichiers de taille acceptable (quelques centaines de kilo-octets). Nous effectuons par ailleurs des traitements numériques originaux qui peuvent être classés en deux catégories : les corrections de type géométrique des données instrumentales et les traitements de type image des projections stéréographiques obtenues.

\section{PRINCIPE DE LA METHODE : MAILLAGE ADAPTATIF.}

Déterminer la figure de pôles \{hkl\} d'un échantillon consiste à localiser, avec le maximum de précision et dans tout le demi-espace défini au-dessus de la surface, les normales aux plans cristallographiques $\{\mathbf{h k l}\}$ existant dans cet échantillon.

En général, par commodité des mesures et aussi pour une plus grande rapidité, il est effectué une exploration hémisphérique pas à pas de ce demi-espace discrétisé en points régulièrement espacés $[2,6,7]$. Pour chaque angle $\psi$ donné, l'angle $\varphi$ est incrémenté par pas $\Delta \varphi=2 \pi / n$ égaux en général aux incréments $\Delta \psi$ qui varient de 0 à $\pi / 2-\eta$; fréquemment $2 \pi / n=5^{\circ}$ et $\eta=5^{\circ}$ si bien que le domaine angulaire exploré correspond à $72 \times 18=1224$ points de mesure (Fig. 2). La figure de pôles classique a donc une durée d'analyse d'environ $20 \mathrm{mn}$, si en chaque point, l'intensité RX est intégrée pendant 1s. Dans le cas d'analyse d'une désorientation fine ou de pôles relatifs à des cristaux de haute qualité (échantillons à gros grains, maclages, etc...), une telle méthode ne devient plus assez précise, car la largeur intrinsèque des pôles est de $1^{\prime}$ 'ordre du $0.01^{\circ}$ et elle fournit des résultats inacceptables. En effet, si l'on diminue le pas d'exploration d'un facteur 100 , le nombre total de pas est $10^{4}$ fois 
plus grand et la durée d'analyse $10^{4}$ fois plus grande (ce qui correspond à plusieurs mois !), sans compter la taille mémoire nour stocker plus de $10^{7}$ mesures.

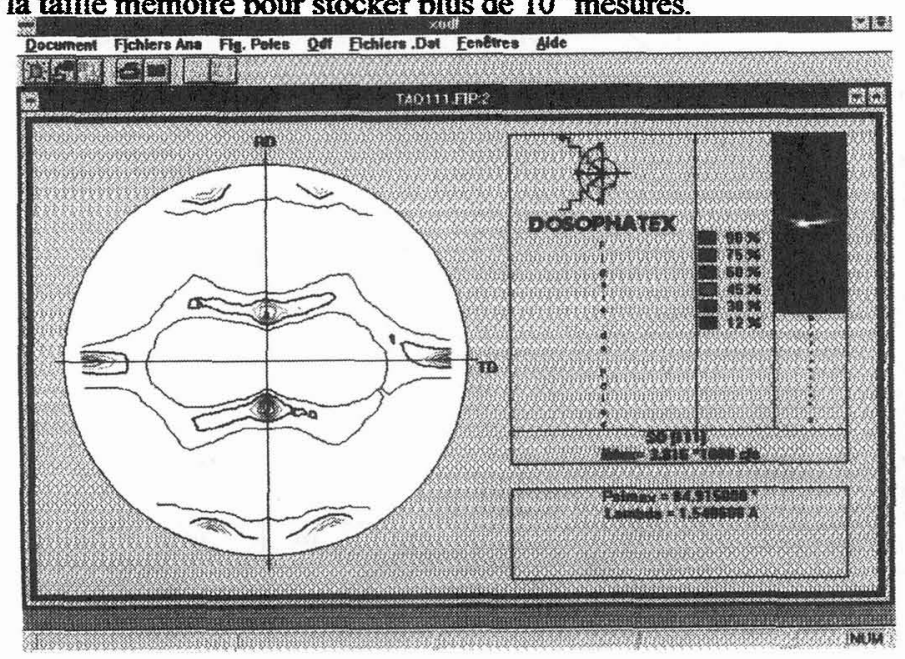

Figure 2: Figure de pôles standard \{111\} d'un échantillon Al. laminé.

Des instrumentations à très haute résolution ont été développées en particulier pour les besoins de caractérisation en micro-électronique sur les couches minces ou les multicouches [8]. Cependant, ces techniques n'ont une très haute résolution de quelques secondes d'arc que selon une direction donnée, ou au plus sur une petite portion de l'espace (quelques degrés). Elles ne peuvent explorer un demi-espace rapidement mais sont utilisées pour des analyses de type "rocking curve" ou des analyses par réflexivité aux petits angles.

Nous avons donc conçu une méthode originale d'analyse qui garde la possibilité d'effectuer une exploration angulaire complète sur une hémisphère et avec une très haute résolution, tout en restant compatible avec un temps de mesure d'environ $20 \mathrm{mn}$. Elle utilise un maillage hémisphérique adaptatif basé sur les deux principes de base suivants [1] :

- Dans le cas de monocristaux ou de très fortes textures, l'intensité RX est diffractée dans un angle solide très étroit; si bien que le maximum est associé à une intensité très forte, tandis que pour une grande partie du domaine exploré, l'intensité est nulle.

- Il existe des relations angulaires entre les différents pôles de la famille \{hkl\} suivant la multiplicité de \{hkl\} et la structure cristallographique du matériau analysé. Compte tenu de la haute résolution et de la largeur des pics \{hkl\}, les pôles de haute multiplicité peuvent être analysés en vue d'une meilleure détermination de leur répartition.

L'exploration sera faite :

- avec un temps d'acquisition très réduit (quelques ms), ce qui permet d'atteindre des vitesses de $360 \%$,

- avec un mode de codage particulier, afin de limiter la place mémoire nécessaire à quelques koctets seulement (au lieu de plus de 10Mo) (Fig. 3). 


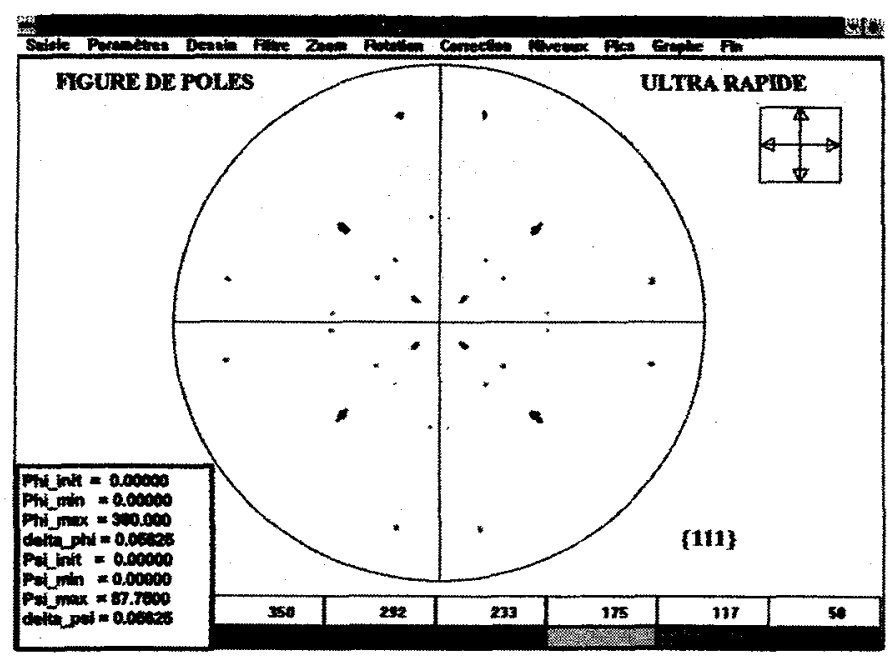

Fiqure 3 : Figure de pôles \{111\} haute résolution d'une couche mince épitaxique GaAs / Ge [100].

L'ensemble de cette analyse de textures à haute résolution est intégré dans le système Dosophatex : grâce à une grande vitesse de rotation et à une acquisition rapide sur une carte Nucleus 2000 , le pas de mesure d'analyse est de $0.01125^{\circ}$ et la durée d'acquisition est de 20 mn environ, tandis que la représentation écran s'effectue en quelques secondes seulement sur un PC486. La seule limitation est que l'intensité du signal diffracté doit être suffisamment forte pour être détectée avec un bon rapport Signal/Bruit en quelques ms; cela nécessite donc une source primaire RX intense avec anode tournante Siemens. Le système de collimation utilisé avec fente Sollers et deux fentes croisées peut être compatible avec une résolution du $1 / 100^{\circ}$, puisque les largeurs des fentes sont réglables; la détection utilise un monochromateur arrière et un détecteur à scintillation. L'ensemble évite ainsi la non-détection de pics de diffraction de dimension inférieure à $1 / 100^{\circ}$ par lissage électronique entre deux pas. Un tel système est bien adapté à l'analyse d'échantillons à gros grains où les diagrammes de diffraction de tous les grains sont enregistrés simultanément (Fig. 4). Il peut aussi s'appliquer à des échantillons à textures moins accentuées comme dans le cas de grandes déformations de monocristaux, en augmentant considérablement la taille des pas d'analyses et le temps d'intégration par pas, ce qui en fait un système universel. 


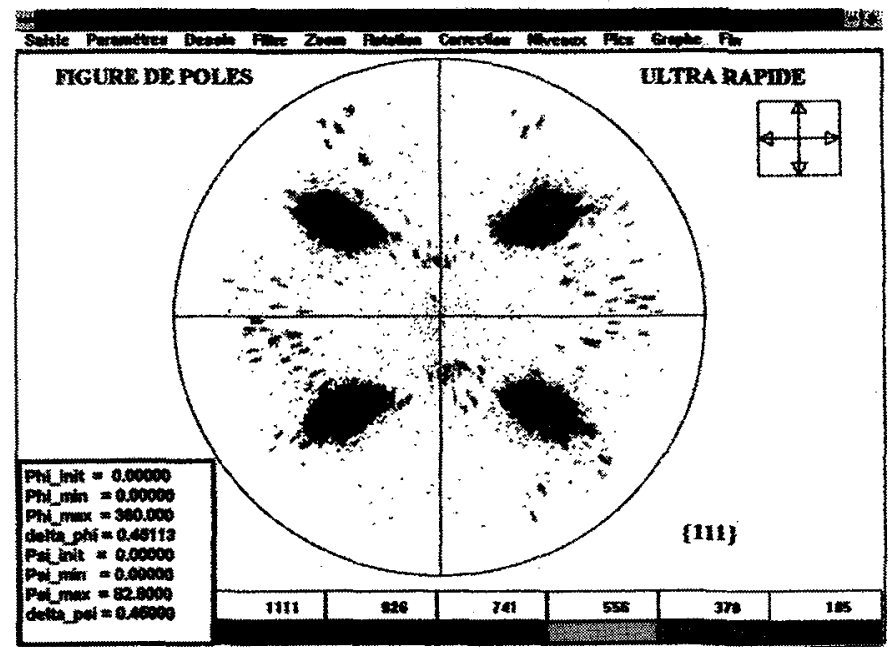

Fiqure 4: Figure de pôles \{111\} d'un échantillon $\mathrm{Al}$ à gros grains.

\section{PRE-TRAITEMENTS DE TYPE GEOMETRIQUE DES DONNEES INSTRUMENTALES}

Un traitement permet de restituer la figure de pôles sous forme de projection stéréographique comme dans le cas classique. Le signal tridimensionnel enregistré est corrigé par une fonction de défocalisation, calculée à la fois d'après les conditions expérimentales (source de rayons $X$, faisceaux $R X$ incidents, détection $R X$ ), la nature de l'échantillon (absorption) et sa forme. Il est aussi possible, dans le cas d'analyse de couches minces, d'effectuer une transformation des coordonnées résultant $d^{\prime}$ une analyse sous incidence rasante $(\alpha-2 \theta$ au lieu de $\theta-2 \theta)$ (Fig. 5 et 6).

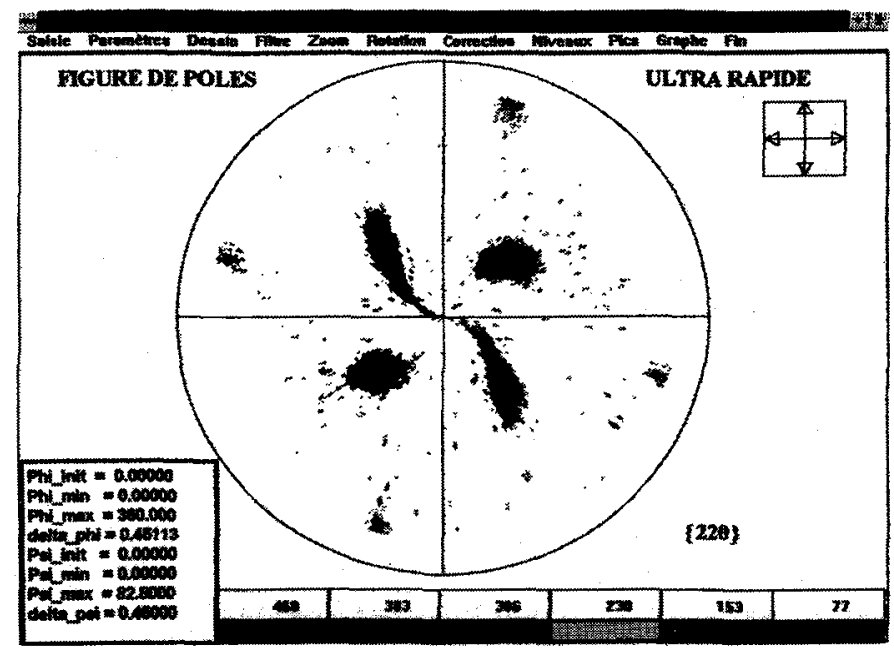

Fiqure 5. Figure de pôles $\{220\}$ d'un échantillon Al à gros grains analyse sous incidence rasante 


\section{TRATTEMENTS DE TYPE IMAGE DES PROJECTIONS STEREOGRAPHIQUES OBTENUES}

L'image est la représentation des valeurs réelles mesurées, quantifiées linéairement ou non selon plusieurs niveaux - ou plusieurs classes -, en rapport avec le maximum absolu après correction. Chaque classe, affichée en fausses couleurs, fait l'objet d'un traitement similaire à l'analyse d'une image en niveaux de gris. Ainsi, tous les traitements locaux (Annexe A.) ou globaux portent sur la valeur quantifiée associée à une couleur, et non sur un quelconque code couleur informatique. Après calcul, on restitue l'image fausses couleurs.

Dans un premier temps, il est nécessaire de séparer les composantes utiles des composantes parasites - ou bruit. Le bruit peut se classer en trois catégories décrites ici dans l'ordre chronologique du traitement :

\subsection{Le traitement du bruit.}

4.1.1 Le bruit de fond.

Il provient essentiellement d'un phénomène d'éclairement (diffusion, fluorescence et rayonnements X parasites environnants), auquel se superpose le signal de diffraction. II peut s'agir d'un fond continu isotrope ou d'un fond variant avec l'orientation.

Une méthode simple consiste à augmenter le seuil de plus bas niveau, ce qui a pour effet d'atténuer les composantes parasites en dessous du seuil. Mais ceci ne s'avère efficace que dans le cas d'un fond à faible dynamique.

Une méthode plus générale [9], valable dans le cas d'un éclairement important, repose sur l'hypothèse que le bruit de fond présente des fluctuations bien moins accentuées que celles du signal utile, et donc qu'il se trouve localisé dans les basses fréquences du spectre. Il s'agit du filtrage homomorphique. Dans ce cas, on applique un filtrage linéaire passe-haut au logarithme du module du signal global. Puis, on reconstitue l'image filtrée par fonction exponentielle. En pratique, ce procédé est surtout efficace pour la localisation des pics fins. Pour les pics larges, il mettra en valeur les frontières, zones où sont concentrées les fluctuations importantes - ou hautes fréquences.

\subsubsection{Les bruits propres à l'affichage.}

Ils se caractérisent par des pixels 'noirs' apparaissant au sein d'un pic qui correspondent à une absence d'information en certaines coordonnées. Nous les appellerons des 'points aberrants'. Ce problème est d'autant plus important que la résolution de l'affichage est grande, pour un nombre de points mesurés constant et dépendant du balayage angulaire. Dans des cas extrêmes, la représentation stéréographique comporte alors des lignes 'noires' provenant de la non-concordance entre une grille angulaire imposée par la mesure (maillage $\varphi$ et $\psi$ ) et une trame rectangulaire (celle de l'affichage). On parlera alors d'effet de 'moiré'. De plus, ce phénomène s'accentue lorsque l'image subit une rotation, puisque certains pixels voisins se trouvent disjoints après projection stéréographique qui ne conserve pas les distances.

Pour remédier à cet inconvénient, nous appliquons une méthode de correction spécifique aux points aberrants, en les remplaçant par des pixels de valeurs calculées à partir de leur voisinage, déterminé par un masque glissant de type $3 \times 3$ (Annexe A). La nouvelle valeur peut être fixée par un facteur de proportion appliqué à la moyenne du voisinage, ou bien, d'une manière plus radicale, elle peut être égale au maximum des pixels voisins. Le résultat est alors une image dont les 'trous' isolés ont été comblés, présentant en contrepartie un élargissement - minime - des frontières (Fig.6). 


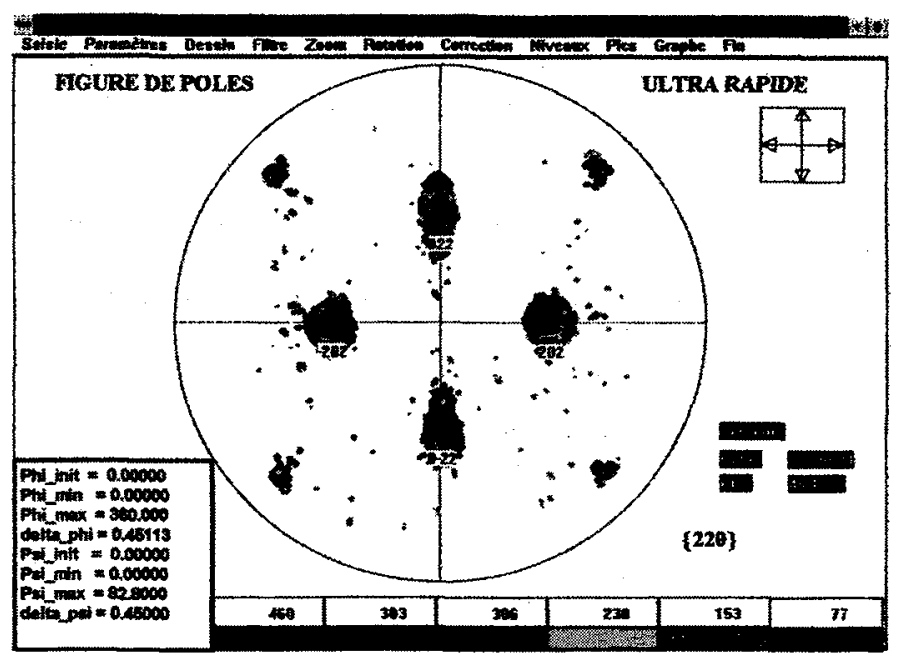

Fiqure 6. Figure de pôles $\{220\}$ après correction d'incidence et correction des points aberrants.

De même que pour la suppression du fond, certains paramètres tels que la dimension et le type du masque de correction sont choisis judicieusement par l'utilisateur, en fonction de l'échantillon analysé.

\subsubsection{Les bruits inhérents au matériau.}

Dans certains cas apparaissent des pixels de poids fort (niveau élevé) autour d'un pic principal. Sachant que le signal mesuré est obtenu par moyennage sur $\mathrm{N}$ échantillons, et que son ergodicité a été vérifiée, nous pouvons en déduire que ces points sont significatifs et sont liés à l'hétérogénéité du matériau. Ainsi, de manière générale, ces points sont sources de fluctuations locales importantes, et par conséquent de hautes fréquences dans le spectre de l'image. II est possible d'effectuer un lissage de l'image obtenue, pour, d'une part reconstituer la continuité des pôles détectés, et d'autre part réduire les fluctuations.

Pour implémenter ce lissage, nous avons à disposition deux types de traitements qui opèrent un filtrage passe-bas de l'image (Cf. figure indicée : Fig. 7) :

- Lissage par masque de convolution.

Il s'agit d'un masque glissant de type $3 \times 3$ (Annexe A) dont les coefficients agissent sur la fréquence de coupure dans le spectre de l'image, le plus commun étant un masque moyenneur (tous les coefficients d'égale valeur). Ce traitement présente l'avantage d'un calcul simple et localisé successivement en chaque point porteur d'information. Il n'y a donc pas de découpage de l'image par blocs. Cependant, il ne permet pas de traiter des bandes de fréquences précises au niveau du spectre.

- Filtrage par transformation de Fourier bidimensionnelle

Il est possible d'isoler une bande de fréquences, et donc de réaliser un filtre passehaut, passe-bande ou passe-bas, ce dernier étant le plus courant. Cette méthode présente donc l'avantage d'un contrôle précis des fluctuations larges et étroites des composantes d'orientations.

\subsection{Quantification}

A ce stade, l'image se trouve corrigée et présente des composantes principales. Nous pouvons donc passer à l'étape suivante du traitement qui consiste à détecter automatiquement ces composantes, à les indicer, puis à déterminer leur orientation ainsi que leur intensité. 


\subsubsection{Orientation des composantes principales.}

La quantification de la texture du matériau impose la connaissance des orientations cristallines \{hkl\}, déduite de la position des pics principaux. Ainsi, l'identification de trois pics au minimum nous donnera sans ambiguité les directions du repère de la représentation stéréographique $(\mathrm{Ox}, \mathrm{Oy}, \mathrm{Oz})$ dans le repère du cristal. Le type de structure du matériau est connu a priori ainsi que le type des pôles \{hkl\} détectés au cours de la mesure (nous supposerons bien sûr que nous sommes dans le cas de la détection d'un seul type de pôles \{hkl\} pour l'angle de Bragg choisi). Nous calculerons ces directions en déterminant la matrice de rotation qui fera coincider les positions des pics théoriques, relatives à une orientation standard du cristal de référence, avec les pics détectés (Fig. 7).

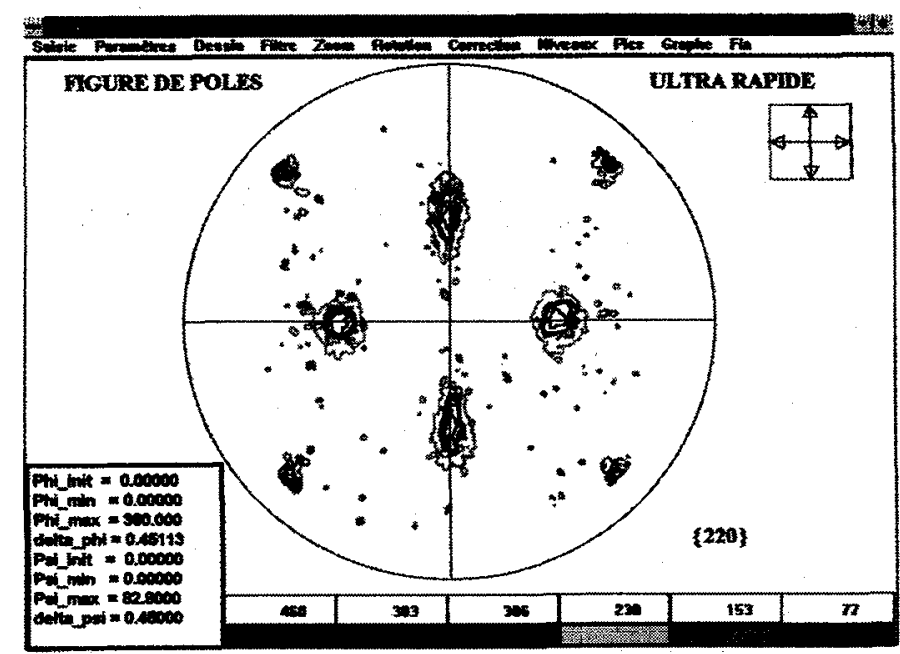

Fifure 7. Figure de pôles $\{220\}$ lissée par masque de convolution $3 \times 3$ et indicee automatiquement

\subsubsection{Quantification par courbes de niveaux.}

La détermination de courbes de niveaux à partir de figures de pôles haute résolution est indispensable pour permettre la quantification des orientations cristallines dans le cas d'orientations très localisées, grâce à des 'passerelles' informatiques entre cette méthode d'analyse adaptative et la nouvelle méthode de calcul des FDOC à haute résolution [5] (Fig. 1).

Après lissage, nous sommes en présence de pôles aux frontières bien définies. Nous mettrons alors en oeuvre une méthode d'analyse hiérarchique [10] travaillant en deux temps : utilisation d'une fonction de répartition bidimensionnelle puis application au résultat d'un masque dérivateur bidirectionnel pour obtenir une fonction de densité bidimensionnelle. Concrètement, le traitement peut s'avérer intéressant pour marquer les frontières d'un seuil ou d'un niveau - donné. La figure 8 représente trois niveaux sur six : 


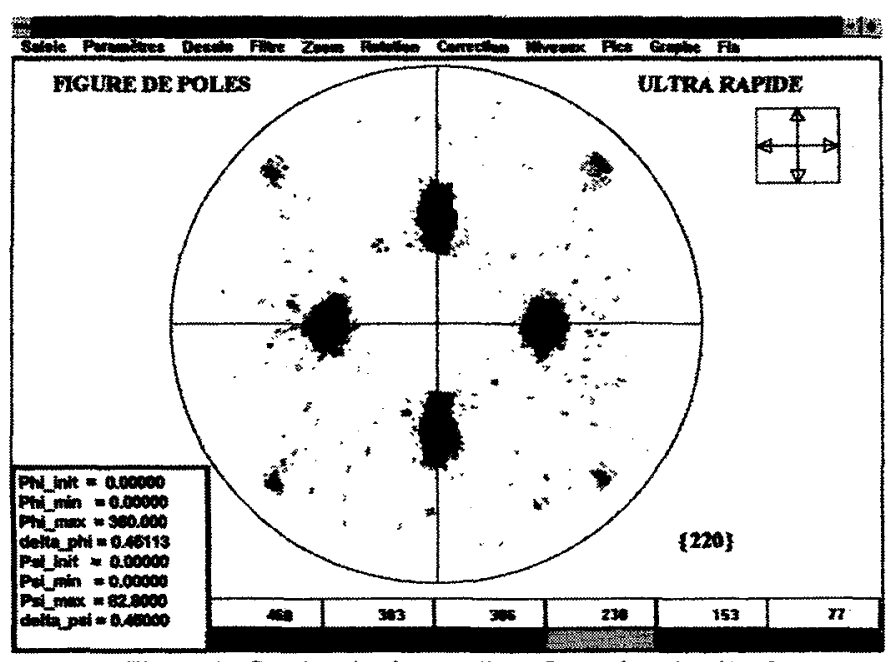

Fiqure 8. Courbes de niveaux d'une figure de pôles $\{220\}$

\section{CONCLUSION}

Ainsi, nous pouvons considérer que nous disposons ainsi d'un outil puissant qui permet une analyse de textures rapide, à la fois par les saisies de données et par les algorithmes utilisés qui reposent sur des fonctions quasi-linéaires du maillage. De plus, cette analyse est non destructive, globale, de haute précision, et complète avantageusement les mesures M.E.T ou E.B.K.D pour les déterminations quantitatives des textures.

[1] FILLIT R.Y. , PERRY A.J., DODELET J.P, PERRIER G., PHILIPPE R. (1991). Nondestructive Characterization of Materials IV pp1-8. New-York. Plenum.

[2] BUNGE H.J. et ESLING C., "Quantitative texture analysis", Deutsche Gesellschaft für Metalkunde, 1982.

[3] VENABLE J.A et HARLAND C.J., "Electron back-scattering patterns, A new technique for obtaining crystallographic information in the scanning electron microscope", Phil. Mag., 1973, 27, 1993-1200.

[4] JUUL JENSEN D. et SCHMIDT N.H., "Local texture measurements by EBSP-New computer procedures and applications", Proc. ICOTOM 9, 1990, 97-102.

[5] FILLIT R.Y. et Al. à paraître.

[6] COULOMB P., "Les textures dans les métaux de réseau cubique", Dunod Paris, 1972.

[7] SCHULZ L.G., J. Appl. Phys., 1949, 20, 1030-1033.

[8] AUVRAY P., CNET Lannion, 1991, communication privée.

[9] JAIN A.K. (1989). Fundamental of Digital Image Processing. Prentice Hall.

[10] DIDAY E., LEMAIRE J., POUGET J., TESTU F., (1982). Eléments d'analyse des données. Dunod. 


\section{Annexe A: masques de traitements semi-locaux.}

Mesque de convolution:

\begin{tabular}{|c|c|c|c|}
\hline & $\mathrm{i}-1$ & $\mathrm{i}$ & $i+1$ \\
\hline$=1$ & A & B & $\mathrm{C}$ \\
\hline & D & $\mathrm{E}$ & $\mathrm{F}$ \\
\hline & $\mathrm{G}$ & $\mathrm{H}$ & I \\
\hline
\end{tabular}

$p($ (ij) : ancienne valeur du point (ij)

$P(i j)$ : nouvelle valeur du point (ij)

$P(i j)=A p(i+1, j+1)+B p(i j+1)+C . p(i-1, j+1)$

+ D.p $(i+1, j)+E p(1, j)+F . p(i-1, j)$

$+\mathrm{Gp}(\mathrm{i}+1, \mathrm{j}-1)+\mathrm{H} \mathrm{Ip}(\mathrm{ij}-1)+\mathrm{H} . \mathrm{p}(\mathrm{i}-1, \mathrm{j}-1)$

Exemple:

Masque

moyennaur:

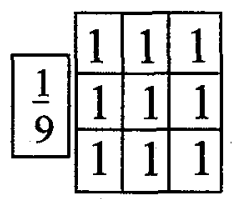

Dérivateur bidirectionnel :

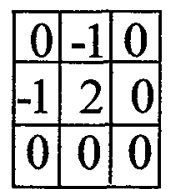

Correction effet de moiré : $\mathrm{P}(\mathrm{i}, \mathrm{j})=\operatorname{Sup}(\mathrm{p}(\mathrm{x}, \mathrm{y}))$, $(i-1<-x<i+1, j-1<-y<j+1)$ 